\title{
Post PCI Systolic Evaluation In Patients With Coronary Artery Disease Using Tissue Doppler Echocardiography
}

\author{
Al Khashab K.A. MD, Ragab T.M. MD, Agiz S.M. M.B.B.Ch \\ 1. Professor of Cardiology and Angiology, Faculty of Medicine Fayoum University. \\ 2. lecturer of Cardiology and Angiology, Faculty of Medicine Fayoum University. \\ 3. Department of Cardiology, Faculty of Medicine Fayoum University.
}

Corresponding author: prof Khaled Ahmed Al Khashab .

\begin{abstract}
:
Background: Patients with stable coronary artery disease may have subtle derangements in their cardiac function before evident changes in conventional echocardiographyderived ejection fraction (EF). These derangements manifest firstly in the longitudinal before circumferential LV function. The effect of PCI on longitudinal and circumferential systolic and diastolic function of the ventricle in patients with chronic stable angina has shown controversial results. In the current study, we tried to evaluate the impact of percutaneous coronary intervention (PCI) on myocardial systolic and diastolic function assessed by different non-invasive indices as EF, Myocardial Performance Index (MPI) and longitudinal myocardial function assessed by Tissue Doppler imaging (TDI) in patients with stable coronary artery disease.
\end{abstract}

Method: A total of 50 patients scheduled for elective PCI were enrolled in this study. All had chronic stable angina and a left ventricular ejection fraction $>55 \%$ with no obvious segmental wall motion abnormality. All underwent conventional echocardiographic evaluation of indices of left ventricular systolic function including EF and MPI, in addition to pulsed-wave tissue Doppler imaging at the mitral and lateral tricuspid annulus. All indices were measured one day before and 5 days after PCI.
Results: $44 \%$ of the patients were males and $56 \%$ were females. The mean age was $55.5 \pm 9.1$ years. Compared to preinterventional values, there was a significant improvement of the mean systolic myocardial velocity $\mathrm{S}^{\prime}$ wave in the $\mathrm{LV}$ as a whole (noticed mainly at the anterior wall) and the lateral RV annulus 5 days after intervention irrespective of the lesion site. Regarding segmental improvement in areas supplied by the lesion targeted by intervention, there was also a highly significant improvement in anterior $\mathrm{S}^{\prime}$ wave velocities 5 days after intervention.

Conclusion: Pulsed wave tissue Doppler is more sensitive in post-PCI monitoring and follow up of patients with chronic stable angina and seemingly normal baseline cardiac function.

Keywords: Tissue Doppler imaging (TDI), Coronary artery disease, Percutaneous Coronary Intervention (PCI), Myocardial Performance Index (MPI).

\section{INTRODUCTION:}

There are various methods, both invasive and non-invasive, for assessing cardiac function. Echocardiography remains central in evaluating cardiac structure and function due to its non-invasive nature, high availability and minimal risk profile. ${ }^{1}$ The most popular echocardiographic method for assessing myocardial contractile function is the 
ejection fraction, which is more of a measure of myocardial circumferential shortening. However, it has been reported that impaired left ventricular longitudinal function may precede circumferential ventricular dysfunction. ${ }^{2}$ Ejection fraction thus may not be an optimal index to detect early derangements in myocardial function.

It was hypothesized that although not evident on standard 2-dimensional echocardiography or contrast ventriculography, reduced regional contractile function distal to coronary artery stenosis may be present in patients with coronary artery disease. ${ }^{1}$

The effect of PCI on systolic and diastolic function in patients with seemingly preserved baseline left ventricular systolic function (by $\mathrm{EF})$ is controversial, and results of echocardiographic assessment of systolic and diastolic ventricular function following successful elective PCI have been contradictory and confusing.

Over the past years, tissue Doppler echocardiography has emerged as a sensitive and quantitative measure of both systolic and diastolic longitudinal myocardial function. ${ }^{4.7}$ It may thus serve as a more suitable index to detect early changes in myocardial function in patients with ischemic heart disease.

\section{AIM OF THE WORK:}

The aim of this study was to determine the impact of percutaneous coronary intervention (PCI) on myocardial systolic and diastolic function assessed by different indices as ejection fraction (EF), "Tei" or Myocardial Performance Index (MPI) and longitudinal myocardial function assessed by Tissue Doppler imaging (TDI) in patients with stable coronary artery disease and seemingly normal baseline EF.

\section{PATIENTS AND METHODS:}

Fifty consecutive patients with coronary artery disease and preserved left ventricular systolic function (EF more than 55\%) who underwent PCI were studied by conventional echocardiography and pulsed wave tissue Doppler imaging at the day of PCI and 5 days thereafter.

\section{Inclusion criteria:}

Patients with stable angina and preserved LV function (by conventional echocardiography) who underwent PCI.

\section{Exclusion criteria:}

1. Recent myocardial infarction or ACS.

2. AF or any ventricular arrhythmias.

3. Previous pacemaker implantation.

4. Dilated cardiomyopathy.

5. Rheumatic heart disease (RHD).

6. Prosthetic valves.

7. Complicated PCI.

8. LVEF less than $55 \%$.

9. Patients whose viability studies showed no significant viable myocardium or scar tissue.

All patients were subjected to the following:

1. Full history taking with emphasis on chest pain and risk factors as diabetes mellitus (DM), hypertension, obesity, cigarette smoking, and family history of premature ischemic heart disease (IHD)

2..12- lead ECG.

3. Echocardiography before PCI as well as five days after intervention. The following measurements and calculations were obtained by using standard views for $\mathrm{M}$ Mode, two dimensional and Doppler studies according to the guidelines of the American Society of Echocardiography: left ventricular ejection fraction (EF) was calculated using left ventricular (LV) end systolic diameter (ESD) and end diastolic diameter (EDD) by using standard M-Mode technique in parasternal long axis view. LV diastolic filling patterns were determined by the mitral inflow pulsed wave Doppler examination. In the apical 4-chamber view, the Doppler sample volume was placed in 
the middle of $\mathrm{LV}$ inflow tract $1 \mathrm{~cm}$ below the plane of mitral annulus between the mitral leaflet tips, where maximal flow velocity in early diastole was recorded. Peak velocities of early (E) and late (A) filling waves were derived from the mitral inflow velocity curve. The ratio of early to late peak velocities (E/A) was calculated. The LV outflow pattern was recorded from the apical 5-chamber view with the pulsed wave Doppler positioned just below the aortic valve. Doppler time intervals of Myocardial Performance Index were measured from mitral inflow and LV outflow velocity as described below:

a) The isovolumic relaxation time (IVRT) was measured from closure of the aortic valve to opening of the mitral valve.

b) The isovolumic contraction time (IVCT) was measured from closure of the mitral valve to opening of the aortic valve.

c) Ejection time (ET) was measured from the opening to the closure of the aortic valve on the LV outflow velocity profile.

d) The Mitral Valve Closure Opening Time (MCOT) was defined as Isovolumic Contraction Time (IVCT) + Ejection Time (ET) + Isovolumic Relaxation Time (IVRT).

e) The Myocardial Performance Index (MPI) or Tei index was calculated as: ${ }^{5}$

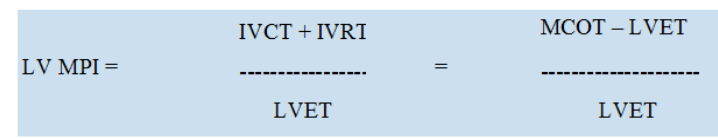

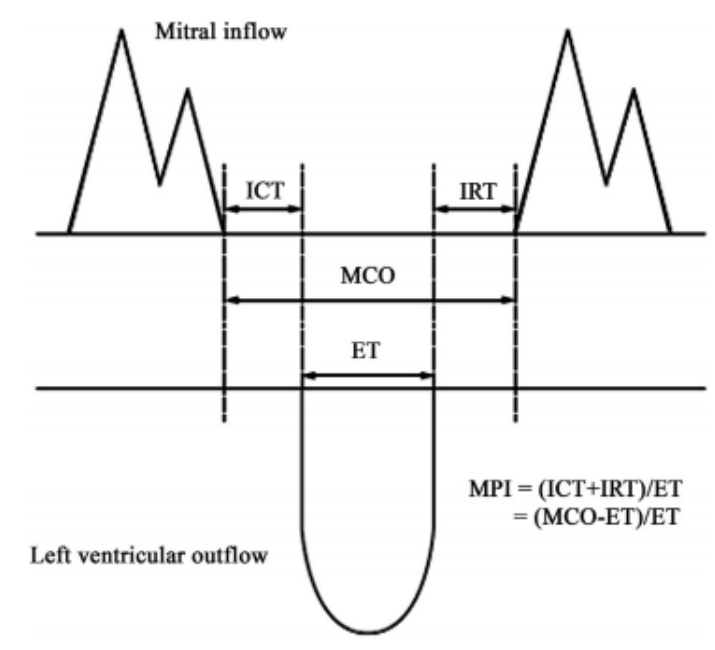

1- Pulsed-wave tissue Doppler echocardiography was done before PCI, as well as 5 days after intervention. Myocardial tissue Doppler velocities were measured (in centimeters per second). The following values were considered normal ${ }^{4.6}$ : 


\begin{tabular}{|c|c|c|}
\hline $\begin{array}{l}\text { Measured } \\
\text { Parameter }\end{array}$ & Males & Females \\
\hline \multicolumn{3}{|c|}{$\begin{array}{l}\text { Mitral Valve Pulsed } \\
\text { Doppler }(\mathrm{cm} / \mathrm{s})\end{array}$} \\
\hline E wave & $0.74 \pm 0.16$ & $0.79 \pm 0.18$ \\
\hline A wave & $0.59 \pm 0.17$ & $0.61 \pm 0.16$ \\
\hline \multicolumn{3}{|l|}{ TDI parameters } \\
\hline \multicolumn{3}{|c|}{ LV Velocities $(\mathrm{cm} / \mathrm{s})$} \\
\hline Septal S' wave & $8.4 \pm 1.4$ & $7.9 \pm 1.3$ \\
\hline Lateral $S^{\prime}$ wave & $10.1 \pm 2.6$ & $9.5 \pm 2.3$ \\
\hline Anterior $\mathrm{S}^{\prime}$ wave & $9.0 \pm 2.2$ & $9.3 \pm 4.9$ \\
\hline Inferior $S^{\prime}$ wave & $9.2 \pm 1.6$ & $8.7 \pm 1.4$ \\
\hline Septal E' wave & $10.1 \pm 3.1$ & $10.3 \pm 2.9$ \\
\hline Lateral $E^{\prime}$ wave & $13.5 \pm 4.0$ & $13.5 \pm 4.1$ \\
\hline Anterior $E^{\prime}$ wave & $11.7 \pm 3.6$ & $12.0 \pm 3.7$ \\
\hline Inferior $E^{\prime}$ wave & $11.3 \pm 3.5$ & $12.2 \pm 3.6$ \\
\hline Septal A' wave & $9.9 \pm 2.0$ & $9.0 \pm 1.9$ \\
\hline Lateral A' wave & $9.5 \pm 2.7$ & $9.0 \pm 2.6$ \\
\hline Anterior $\mathrm{A}^{\prime}$ wave & $8.8 \pm 2.2$ & $8.2 \pm 2.2$ \\
\hline Inferior $\mathrm{A}^{\prime}$ wave & $10.5 \pm 2.3$ & $9.8 \pm 2.3$ \\
\hline \multicolumn{3}{|c|}{ RV Velocities $(\mathrm{cm} / \mathrm{s})$} \\
\hline$S^{\prime}$ wave & $13.6 \pm 2.8$ & $13.1 \pm 2.1$ \\
\hline$E^{\prime}$ wave & $12.8 \pm 3.8$ & $13.4 \pm+3.7$ \\
\hline $\mathrm{A}^{\prime}$ wave & $12.9 \pm 3.8$ & $13.2 \pm 4.0$ \\
\hline
\end{tabular}

least one year after PCI. Unfractionated heparin (10,000 units, I.V.) was given during

The sample volume was taken at; septal, lateral, anterior, and inferior angles of mitral annular ring, and also at lateral angle of tricuspid valve annulus.

In addition, pulsed-wave Doppler mitral and tricuspid inflow velocities, including $\mathrm{E}$ and $\mathrm{A}$ -wave peak velocities (in centimeters per second), were obtained from the apical 4chamber view.

2- Coronary angiography and PCI were performed in a single session at Fayoum University Hospital. All patients received Aspirin 75-100 mg once daily, clopidogrel $75 \mathrm{mg}$ daily starting at least two days before PCI. Aspirin would be continued indefinitely and clopidogrel would be continued for at
PCI in addition to conventional treatment.

3- $\quad$ Patients were followed up for 5 days for the following to be excluded:

a) In hospital acute and sub-acute stent thrombosis.

b) In hospital bleeding.

c) Major adverse cardiac events (MACE), as sudden death, acute myocardial infarction and repeat revascularization.

\section{STATISTICAL ANALYSIS}

Data were collected and coded to facilitate data manipulation and double entered into Microsoft Access and data analysis was performed using SPSS software version 18 in windows 7. Simple descriptive analysis in the form of numbers and 
percentages for qualitative data, and arithmetic means as central tendency measurement, standard deviations as a measure of dispersion for quantitative parametric data, and inferential statistic test were done:

For quantitative parametric data:

- Paired t-test in comparing two dependent quantitative data.

For quantitative non-parametric data

- Mann-Whitney test in comparing two independent groups.

For qualitative data

- Mc-Nemar test for paired dependent qualitative data.

A p-value $\leq 0.05$ was considered the cut-off value for significance.

\section{RESULTS:}

\section{Patient Characteristics}

The study included fifty consecutive patients. $44 \%$ of the patients were males and $56 \%$ were females. Their mean age was 55.5 \pm 9.1 years. They all underwent elective PCI at the Cardiology Department of Al Fayoum University Hospital. $50 \%$ of the patients were diabetic, $62 \%$ were hypertensive, $48 \%$ were smokers, $68 \%$ were dyslipidaemic, $62 \%$ had a positive family history for premature coronary artery disease, and $6 \%$ had previous PCI.

\section{Coronary Angiography and PCI data}

Of the 50 patients enrolled, single-vessel disease was present in 29 patients (divided as follows: 16 patients with an LAD lesion [58\% of the study population], 7 patients with an LCX lesion [14\%], and 6 patients with an RCA lesion [12\%]), 2-vessel disease in 19 patients (38\% of the study population), and 3-vessel disease in 2 patients (4\% of the study population). Forty patients had intervention for all lesions, while 10 had intervention to some of these lesions. In total, 37 interventions were performed (17 in LAD territory, 11 in LCX territory, and 7 in RCA territory).

Effect of PCI on Left Ventricular function by Conventional Echocardiography (Table 1):

The improvement was insignificant between echocardiographic measurements at baseline and 5 days after intervention. The mean value of ejection fraction obtained by M- mode before PCI was 0.647 , while five days after PCI it became 0.648. Diastolic function obtained by trans-mitral inflow also showed no significant difference 5 days after PCI from the baseline.

Table 1: Comparison of Conventional Echo findings before and after Catheterization among the study group.

\begin{tabular}{|c|c|c|c|c|c|c|}
\hline \multirow{2}{*}{ Variable } & \multicolumn{2}{|c|}{ Before } & \multicolumn{2}{|l|}{ After } & \multirow{2}{*}{ p-value } & \multirow{2}{*}{ Sig. } \\
\hline & Mean & SD & Mean & SD & & \\
\hline EF\% & 0.647 & 0.054 & 0.648 & 0.057 & 0.9 & NS \\
\hline \multicolumn{7}{|c|}{ Diastolic function } \\
\hline Normal & 23 & $46 \%$ & 20 & $40 \%$ & \multirow{2}{*}{0.5} & \multirow{2}{*}{ NS } \\
\hline Grade I & 27 & $54 \%$ & 30 & $60 \%$ & & \\
\hline
\end{tabular}

Using Myocardial performance (Tei) index as an indicator for Left ventricular improvement in patients with chronic stable angina (Table 2): 
Tei index obtained from conventional echocardiography showed no significant improvement 5 days after PCI from the Effect of PCI on Left Ventricular Function using Pulsed Tissue Doppler (Tables 3, 4, 5):

There was a significant improvement in the mean systolic function of the left ventricle in general and the anterior wall segment in specific, with a p-value $<0.05$ in systolic $\mathrm{S}$-wave of the anterior segment and the mean $\mathrm{S}$ wave. There was also a significant improvement in right ventricular systolic function when measured by pulsed baseline, with a p-value $>0.05$. The mean value before PCI was (0.41), while the mean value after 5 days was (0.4). tissue Doppler at the lateral tricuspid annulus, with a p-value $<0.05$.

On observing the lesion site in relation to its corresponding myocardial segment, we found that there was a significant improvement in the $S$ wave of the anterior wall of the left ventricle 5 days after LAD intervention, with a p-value $<0.05$. However, there was no significant improvement in the diastolic LV function (whether $\mathrm{E}$ or $\mathrm{A}$ waves).

Table (2): Comparison of Myocardial Performance Index before and after Catheterization among study group.

\begin{tabular}{|l|l|l|l|l|l|l|}
\hline \multirow{2}{*}{ Variables } & \multicolumn{2}{l|}{ Before } & \multicolumn{2}{l|}{ After } & \multirow{2}{*}{ p-value } & Sig. \\
\cline { 2 - 6 } & Mean & SD & Mean & SD & S. & NS \\
\hline $\begin{array}{l}\text { Myocardial } \\
\text { performance } \\
\text { index }\end{array}$ & 0.41 & 0.05 & 0.40 & 0.06 & 0.4 & \\
\hline
\end{tabular}

Table (3): Comparison of Pulsed Tissue Doppler systolic S-wave of different ventricular segments before and after catheterization among study group.

\begin{tabular}{|c|c|c|c|c|c|c|}
\hline \multirow{2}{*}{$\begin{array}{l}\text { Systolic } \\
\text { S-wave }\end{array}$} & \multicolumn{2}{|c|}{ Before } & \multicolumn{2}{|l|}{ After } & \multirow{2}{*}{ p-value } & \multirow{2}{*}{ Sig. } \\
\hline & Mean & SD & Mean & SD & & \\
\hline \multicolumn{7}{|l|}{ Mitral valve } \\
\hline Lateral & 10.56 & 2.6 & 10.94 & 2.97 & 0.6 & NS \\
\hline Septum & 9.38 & 2.7 & 9.6 & 2.1 & 0.5 & $\mathrm{NS}$ \\
\hline Anterior & 9.26 & 3.2 & 10.02 & 2.3 & 0.01 & $\mathbf{S}$ \\
\hline Inferior & 9.86 & 2.3 & 10.1 & 1.9 & 0.3 & NS \\
\hline Mean S-wave & 9.76 & 2.4 & 10.17 & 2.03 & 0.01 & $\mathbf{S}$ \\
\hline \multicolumn{7}{|c|}{ Tricuspid valve } \\
\hline Lateral & 12.92 & 3.9 & 14.08 & 4.3 & 0.01 & $\mathbf{S}$ \\
\hline
\end{tabular}


Table (4): Comparison of Pulsed Tissue Doppler diastolic E-wave of different ventricular segments before and after catheterization among study group.

\begin{tabular}{|c|c|c|c|c|c|c|}
\hline \multirow{2}{*}{$\begin{array}{l}\text { Diastolic } \\
\text { E-wave }\end{array}$} & \multicolumn{2}{|l|}{ Before } & \multicolumn{2}{|l|}{ After } & \multirow{2}{*}{ p-value } & \multirow{2}{*}{ Sig. } \\
\hline & Mean & SD & Mean & SD & & \\
\hline \multicolumn{7}{|l|}{ Mitral valve } \\
\hline Lateral & 10.26 & 4.2 & 10.52 & 4 & 0.4 & NS \\
\hline Septum & 7.86 & 3.7 & 7.58 & 3 & 0.9 & NS \\
\hline Anterior & 8.52 & 3.7 & 8.88 & 3.1 & 0.09 & NS \\
\hline Inferior & 8.12 & 3.4 & 8.26 & 2.7 & 0.4 & NS \\
\hline Mean S-wave & 8.69 & 3.29 & 8.79 & 2.9 & 0.2 & NS \\
\hline \multicolumn{7}{|c|}{ Tricuspid valve } \\
\hline Right & 8.18 & 3.3 & 8.30 & 3 & 0.5 & NS \\
\hline
\end{tabular}

Table (5): Comparison of Pulsed Tissue Doppler diastolic A-wave of different ventricular segments before and after catheterization among study group.

\begin{tabular}{|l|l|l|l|l|l|l|l|}
\hline \multirow{2}{*}{$\begin{array}{l}\text { Diastolic } \\
\text { A-wave }\end{array}$} & Before & After & p- & \multirow{2}{*}{ Sig. } \\
\cline { 2 - 7 } Mitral valve & Mean & SD & Mean & SD & value & \\
\hline Lateral & 10.88 & 4.1 & 10.86 & 4.2 & 0.3 & NS \\
\hline Septum & 10.28 & 4 & 9.82 & 3.2 & 0.9 & NS \\
\hline Anterior & 10.14 & 5.6 & 9.82 & 3.4 & 0.9 & NS \\
\hline Inferior & 10.7 & 3.2 & 11.12 & 3.3 & 0.1 & NS \\
\hline Mean S-wave & 10.5 & 3.8 & 10.41 & 3 & 0.4 & NS \\
\hline Tricuspid valve & 13.84 & 5.9 & 14.48 & 5.9 & 0.2 & NS \\
\hline Lateral & \multicolumn{7}{|l|}{} \\
\hline
\end{tabular}




\section{Discussion:}

The aim of this study was to determine the impact of PCI on myocardial systolic and diastolic function assessed by different indices as ejection fraction (EF), myocardial performance index (MPI) and longitudinal myocardial function assessed by tissue Doppler imaging (TDI) in patients with chronic stable coronary artery disease.

Fifty consecutive patients with coronary artery disease and preserved left ventricular systolic function (EF more than 55\%) who underwent PCI were studied by conventional echocardiography and pulsed wave tissue Doppler imaging at the day of PCI and 5 days after PCI.

In our study there was no significant improvement in LV systolic function (represented by EF) or diastolic function (obtained by trans-mitral inflow) five days after PCI. There was also no significant reduction in the mean MPI of all groups. However, there was a significant improvement in the tissue Doppler derived mean and anterior wall LV systolic myocardial velocity ( $\mathrm{S}$ wave) in addition to the lateral $\mathrm{RV}$ wall $\mathrm{S}$ wave 5 days after intervention, irrespective of the lesion site. The rest of the LV walls showed no significant improvement in the $\mathrm{S}$ wave. There was also no significant effect on diastolic $\mathrm{E}$ and $\mathrm{A}$ waves in both mitral and tricuspid valves after intervention.

To assess whether improvement of left ventricular function was related to revascularization, myocardial velocities before and after PCI were compared in areas supplied by the lesion targeted by intervention. We found a highly significant improvement in anterior $S$ wave velocities 5 days after intervention to $\mathrm{LAD}$, while there was no significant improvement noted in the diastolic waves of the anterior wall nor in the systolic and diastolic waves of the remaining walls.

When comparing our study to previous studies we found points of agreement and some other points of disagreement. This might be due to several reasons.

Rashid et al, $2014^{10}$ examined fifty patients with STEMI, who underwent primary PCI. Echocardiography with DTI was performed for all patients at baseline and one month after PCI. They found a significant improvement in the LV EF, mean $\mathrm{S}$ wave and mean $\mathrm{E}$ wave of successfully reperfused patients after PCI $(\mathrm{p}<0.01)$. They showed no significant correlation between the mean values of A wave at baseline and after PCI ( $p$ $>0.05$ ). Obviously, their study group (STEMI patients) was different from ours.

Nozari et al, $2012^{11}$ examined 115 patients with CAD who underwent elective PCI. They did not put a limit to ejection fraction and they excluded patients with a normal echo study. All patients had TDI and conventional echo done before, one day after PCI and 3-6 months later. They demonstrated that EF significantly increased during the 3 stages of measurements $(\mathrm{P}<0.0001)$. Diastolic dysfunction significantly improved the day after PCI ( $p<0.0001)$, but no further changes were seen in the results 3-6 months later. Again, in this study, the studied group was different from ours, as they excluded patients with a normal ejection fraction.

Rashid et al, $2012{ }^{8}$ examined 25 consecutive patients with chronic stable angina and preserved LV systolic function (EF > $50 \%$ ) who underwent PCI. All were studied by pulsed wave tissue Doppler before PCI as well as 1 day and 6 weeks after intervention to evaluate systolic and diastolic function. Regarding their results, they agreed with our study in showing no significant improvement in all conventional echocardiographic measures at baseline, 1 day, and 6 weeks after intervention. They also showed a significant improvement in the $S$ wave of the anterior wall of the LV and lateral wall of the RV 1 day and 6 weeks after intervention. However, they disagreed with our study by showing a significant increase in systolic myocardial velocity $S$ wave at other walls (inferior, septal, and lateral). They also showed a significant increase in trans-mitral and trans-tricuspid early and late diastolic myocardial velocities after intervention. The reason might be related to successful PCI done for all significant stenotic lesions in their study. In our study, on the other hand, PCI was done to the most significant stenotic lesions, leaving some residual lesions that still required further PCI 
sessions. In addition, the number of patients in their study may have been too small to reach a statistically significant conclusion.

Diller et al, $20099^{9}$ examined 24 consecutive patients with chronic stable angina and preserved systolic LV function who underwent elective PCI. Transthoracic echocardiography and tissue Doppler imaging were performed before PCI as well as 1 day and 6 weeks after intervention to evaluate systolic and diastolic function. Their results agreed with ours in showing that ejection fraction, trans-mitral and trans-tricuspid flow Doppler parameters failed to reflect any improvement 1 day and 6 weeks after PCI. (7) The right ventricle also showed significant improvement in tissue Doppler measures of systolic function. However, their results disagreed with ours in many other aspects. They showed significant improvement of the systolic myocardial peak velocities after PCI in the septal, lateral, and inferior walls and an insignificant trend toward an improvement in the posterior wall. There was a significant increase in tissue Doppler measures of early and late diastolic function at 1 day and 6 weeks after PCI. The reason might also be related to successful PCI done for all significant stenotic lesions in their study and relatively longer period of follow up. The number of patients in their study may have also been too small to reach a statistically significant conclusion.

It was noted that there was a significant improvement in $\mathrm{RV}$ function regardless of the artery tackled by PCI (in RCA and non-RCA lesions). This may be explained by the "ventricular interdependence" theory. Both ventricles are enclosed within the same pericardial sac, are supported by the same fibrous skeleton, are encircled by common muscle fibers and share the same interventricular septum. Systolic dysfunction of the LV can affect RV function through IVS dysfunction, annular/fibrous skeleton interaction, or pericardial tethering/interaction.

\section{CONCLUSION:}

In our study, we found that mean and anterior wall systolic function measured by TDI improved 5 days after successful PCI. This affirms the idea that there may be an unnoticed reduction in contractile function in patients with chronic stable angina, that could very possibly improve after successful revascularization. The longitudinal LV contractile function (represented by mitral annular motion) shows earlier affection and recovery than the circumferential function (represented by the classical EF). This suggests that pulsed wave tissue Doppler, an easily derived index of LV longitudinal function, is more sensitive than ejection fraction in monitoring and follow up of patients with stable angina and seemingly normal baseline cardiac function.

On the other hand, diastolic function results obtained by tissue Doppler, or through trans-mitral and trans-tricuspid flow velocities by traditional measures failed to reflect any improvement in diastolic function. Furthermore, myocardial performance index didn't add any benefit that may serve patients with chronic stable angina.

Finally, further studies on hibernating myocardium are needed to estimate the actual time needed by the myocardium to restore its normal contractility and if it is affected by the duration of ischemia to further clarify the role and sensitivity of TDi in evaluating ischemia in CAD patients.

\begin{tabular}{|l|l|}
\hline LIST OF ABBREVIATION \\
\hline Subject & Meaning \\
\hline DM & Diabetes mellitus \\
\hline EF & Ejection fraction \\
\hline ECG & Electrocardiogram \\
\hline HF & Heart failure \\
\hline HTN & Hypertension \\
\hline IHD & Ischemic heart disease \\
\hline LAD & Left anterior descending \\
\hline LCX & Left circumflex \\
\hline LVEF & Left ventricular ejection fraction \\
\hline RV & Right ventricle \\
\hline RCA & Right coronary artery \\
\hline PCI & $\begin{array}{l}\text { Percutaneous } \\
\text { intervention }\end{array}$ \\
\hline TDI & Tissue Doppler imaging \\
\hline
\end{tabular}

\section{REFERENCES:}

1. Michael Ruisi, Michael Levine, Dennis Finkielstein.The Assessment and Potential Implications of the Myocardial Performance 
Index Post Exercise in an at Risk Population.2013. Cardiol Res. 2013;4(6):173177

2. Yukio Mizuguchi MD, Yukio Mizuguchi MD, Yoshifumi Oishi, et al, The Functional Role of Longitudinal, Circumferential, and Radial Myocardial Deformation for Regulating the Early Impairment of Left Ventricular Contraction and Relaxation in Patients With Cardiovascular Risk Factors: A Study With Two-Dimensional Strain Imaging, , 2008. DOI: https://doi.org/10.1016/j.echo.2008.07.0 $\underline{16}$

3. Islam Galal. Improvement Of Regional Myocardial Function In Patients With Coronary Artery Disease Before And After Coronary Intervention By Tissue Doppler Study. 2013 http://www.publications.zu.edu.eg/Pages/PubS how.aspx?ID=20410\&\&pubID=19

4. Navtej S. Chahal, Tiong K. Lim, Piyush Jain, et al, Normative reference values for the tissue Doppler imaging parameters of left ventricular function: a population-based study, 2009. European Journal of Echocardiography (2010) 11 , $51-56$ doi:10.1093/ejechocard/jep164.

5. Tei C, Nishimura RA, Seward JB, Tajik AJ. Noninvasive Doppler-derived myocardial performance index: correlation with simultaneous measurements of cardiac catheterization measurements. J Am Soc Echocardiogr 1997; 10: 169-178.
6. Alam M, Wardell J, Nordlander R, et al . Characteristics of mitral and tricuspid annular velocities determined by pulsed wave Doppler tissue imaging in healthy subjects. 1999. J Am Soc.Echocardiogr; 12: 618-28

7. Wang J., Abraham T.P., Belohlavek M, et al. Delayed onset of subendocardial diastolic thinning at rest identifies hypoperfused myocardium. 2005.

8. Hesham Rashid, Ahmed Abdel-Moniem , Sameh Emai, et al, Evaluation Of Myocardial Function In Patients With Chronic Stable Angina And Apparent Normal Ventricular Function (Tissue Doppler Study Before And After PCI) .2012. Heart Mirror J 2012; 6(2): 141-147.

9. Gerhard-Paul Diller, Balvinder S, Simon A, et al. 2009: Patients with Chronic Stable Angina and Apparent Normal Ventricular Function-A Tissue Doppler Study Before and After PCI. J Am Soc Echocardiodr; volume 22, issue 2.

10. Rashid H, El-Enien HA, Ibraheem M, et al. The Predictive Value of Tissue Doppler for Left Ventricular Recovery and Remodeling after Primary Percutaneous Coronary Intervention.2014. J Cardiol Curr Res 1(6): 00032. DOI: $10.15406 /$ jccr.2014.01.00032

11. Younes Nozari1, Nader Jangi Oskouei, Zahra Khazaeipour, et al. Effect of Elective Percutaneous Coronary Intervention on Left Ventricular Function in Patients with Coronary Artery Disease.2011. Acta Medica Iranica, 2012; $\quad 50(1)$ : 26-30. 